\title{
Frame rate required for speckle tracking echocardiography: A quantitative clinical study with open-source, vendor-independent software.
}

Short title: FPS

Madalina Negoita, PhD, a

Massoud Zolgharni, BSc, MPhil, PhD, a

Elham Dadkho, BSc, a

Matteo Pernigo, MD, b

Michael Mielewczik, Dr. sc. ETH Zurich, a

Graham Cole, MA MRCP, a

Niti M. Dhutia, MEng, PhD, a

Darrel P Francis, MA MRCP, a

a. Imperial College London, National Heart and Lung Institute, Hammersmith Hospital Campus, London W12 ONN

b. University of Verona, Department of Medicine, Section of Cardiology

Correspondence:

ICTEM Building Level 3

National Heart and Lung Institute

Imperial College London

Hammersmith Hospital Campus

Du Cane Road

London W12 0NN

UK

Tel: +44 2075941093

Fax: +44 2080825109

Email: m.negoita@imperial.ac.uk

Word count: 4207

Keywords

Speckle Tracking, Frame Rate, Myocardial Velocity, Sampling Frequency, Tissue Velocity Imaging 


\section{Abstract}

Objectives: To determine the optimal frame rate at which reliable heart walls velocities can be assessed by speckle tracking.

Background: Assessing left ventricular function with speckle tracking is useful in patient diagnosis but requires a temporal resolution that can follow myocardial motion. In this study we investigated the effect of different frame rates on the accuracy of speckle tracking results, highlighting the temporal resolution where reliable results can be obtained.

Material and methods: 27 patients were scanned at two different frame rates at their resting heart rate. From all acquired loops, lower temporal resolution image sequences were generated by dropping frames, decreasing the frame rate by up to 10 -fold.

Results: Tissue velocities were estimated by automated speckle tracking. Above 40 frames/s the peak velocity was reliably measured. When frame rate was lower, the inter-frame interval containing the instant of highest velocity also contained lower velocities, and therefore the average velocity in that interval was an underestimate of the clinically desired instantaneous maximum velocity.

Conclusions: The higher the frame rate, the more accurately maximum velocities are identified by speckle tracking, until the frame rate drops below 40 frames/s, beyond which there is little increase in peak velocity. We provide in an online supplement the vendor-independent software we used for automatic speckle-tracked velocity assessment to help others working in this field. 


\section{Introduction}

Echocardiography is commonly used to assess myocardial motion to evaluate heart dysfunction. Two-dimensional speckle tracking is a newer tool for analysing echocardiographic images. The technique is based on tracking echo-dense speckles within the myocardial tissue on sequences of consecutive frames [1-4].

Speckle-tracked velocity estimates are dependent on the acquisition frame rate, because velocities can change rapidly. Clinicians want to measure the instantaneous peak velocity, but if between two acquired frames, a piece of tissue accelerates to a brief peak velocity and then decelerates again, the speckle tracking algorithm can only see the total distance moved during that time and divide by the time interval, and therefore report the average velocity during that interval. If the acceleration and deceleration during that interval is sharp, this average velocity during the interval will be an underestimate of the instantaneous peak velocity.

A variety of desirable rates have been recommended from 40 to 80 frames/s [1, 2, 5-9] for normal heart rates, but there appear to be no studies specifically investigating how the desirable frame rate depends on heart rate and quantitatively what the consequences are of using a lower frame rate.

In this study, we examined the relationship between myocardial tissue velocities estimated by speckle tracking and frame rate of image sequences used for analysis. 


\section{Material and methods}

\subsection{Image Acquisition}

To test the limits of adequate frame rates, which allow reliable estimations of tissue wall velocities, we acquired B-mode apical 4-chamber view image sequences (Figure 1a). Image sequences were acquired for 20 seconds with a resolution of $640 \times 480$ pixels per frame and a sampling rate of 8-Bits per frame (grayscale). The acquisition machine used for this study is GE Vivid-I (General Electric, Hatfield, UK).

Image sequences were stored as DICOM files and then exported for offline analysis. Throughout image acquisition, the scanning depth and frequency of the transducer remained fixed. We acquired at frame rates of 52 and 79 frames/s by adjusting the sector width until the machine was acquiring at these desired frame rates.

\subsection{Patients}

27 patients (10 males and 17 females) aged between 25 and 89 were scanned for two different frame rates 52 respectively 79 frames/s on their base heart rate. Patients had electrocardiogram electrodes connected throughout the acquisition. The image sequences were recorded consecutively, with the probe kept in one position for both frame rates.

Our protocol was to acquire images, even if image quality was poor, so that we could describe the representativeness of our sample across the generality of patients. We acquired images from 
27 patients but had to abandon the data from two of them because of poor image quality, leaving the 25 taken forward for the analysis.

Patients were approached in an outpatient setting in St Mary's Hospital London without regard to their physical characteristics and by staff unaware of any prior echocardiographic data. All patients who agreed to participate and gave written informed consent then underwent the study. The scans were performed by experienced operators working in St Mary's Hospital Echocardiography Department. The study was approved by the local ethics committee.

\subsection{Simulation of Lower Frame Rates}

To simulate lower frame rates from the acquired image sequences, frames were sub-selected by the pull-down scheme shown in Figure 2. In brief, one in every n frames was sub-selected, with values of $n$ from 2 to 10 . There is more than one choice of which set of 1 -in-n frames to take, as shown in Figure 2 for $n=2$ and $n=3$. We ran each of the possible sets separately and averaged the results of all of them to give the overall result for that value of $n$. This process allowed us to achieve up to a 10 -fold reduction of frame rate. This was performed offline, using Matlab R2013a (Mathworks Inc.).

Using this process of frame rate reduction by up to 10 -fold, and raw acquired data of 52 frames/s and 79 frames/s, we obtained for each patient 20 different frame rates, covering a wide range from 5 frames/s to 79 frames/s. All 20 resulting datasets for each patient were then treated in the same way.

\subsection{Velocity Estimation by Speckle Tracking}


For each patient, acquired and constructed datasets at 20 different frame rates, each underwent speckle tracking separately. The region of interest, highlighted by the white box in Figure 1a, within which tissue velocities were computed, was selected manually to remove errors introduced by selecting a different position automatically. This region was kept constant for all 10 frame rates, which were originating from a single raw acquired frame rate (i.e. the raw acquired frame rates and all 9 constructed lower frame rates).

To make as much of the data to be analysable as possible, this study addressed the septal wall, whose position and better contrast make it more consistently trackable, and whose lateral motion is relatively small so that the tissue does not leave the echocardiographic region of interest during the cardiac cycle. The vertical movement of the tissue was used to construct the velocity curves (Figure 1b black curve). The values for peak systolic wave (S wave - Figure $1 \mathrm{~b}$ red squares) and peak early (E wave - Figure $1 \mathrm{~b}$ green diamonds) and late (A wave - Figure 1b blue circles) diastolic waves were extracted and averaged across all beats for each sequence.

At very low frame rates, the algorithm often was unable to track speckles and therefore no velocity was obtained. This often happened for frame rates below 10 frames/s.

Online appendix A shows all the data for each individual patient separately. For each patient and each of the 20 frame rates, the mean s' peak velocity, mean e' peak velocity and mean a' peak velocity for that image sequence (of several beats) were obtained, as well as the individual beat-by-beat values. The mean values are plotted in the figures as black points. 


\section{Results}

\subsection{Single example patient}

Figure 3 shows the results from a single patient. The upper panel shows the 20 measured peak s' wave velocities, each using data of a different frame rate, from a patient whose heart rate is $64 \mathrm{bpm}$. The measured peak s' wave velocities are approximately $4.7 \mathrm{~cm} / \mathrm{s}$ for all 5 frame rates above 20 frames/s. At progressively lower frame rates, however, the measured s' peak velocity falls towards zero rapidly. At 10 frames/s it is already reduced by half. The middle and lower panels (Figure 3b and 3c) show a similar pattern for the e' and a' velocities, although in both cases the velocity begins to decline at approximately 40 frames/s.

The corresponding individual patient data for all 25 patients are shown in appendix A. The reason for lower frame rates giving lower velocities, especially for the e' and a' waves, is given in Figure 4. The panels show 3 velocity traces, from image data at 79 (continuous black line -), 20 (dashed red line - - ) and 11 (dotted blue line $\cdot-\cdot)$ frames/s respectively. The traces are smoothed with a Savtsky-Golay filter of $3^{\text {rd }}$ polynomial degree and 11 points on the upper panel (Figure 4a) used for extracting the s' wave while the lower panel (Figure 4b) uses a Savtsky-Golay filter of $5^{\text {th }}$ polynomial degree and 7 points for extracting the faster timeoccurring e' and a' waves. The filters are used for removing noise in the velocity curve.

At the highest frame rate, the full shape of s', e' and a' is correctly identified for each corresponding filter. At the lower rate of 20 frames/s, while the s' velocity peak is still adequately identified, the e' and a' waves being brief in duration, are inadequately tracked, and their peak velocities therefore underestimated. At the lowest frame rate shown, 11 frames/s, 
tracking of movement is inadequate for all three waves. Because the durations of the e' and a' peaks are shorter, the frames need to be closer in time to adequately capture their peak velocity.

Online appendix B shows, with data from one patient that it does not matter which set of one in two frames (odd or even), is used for analysis. Also, the online appendix B shows this for the three possibilities of a 3-to-1 frame rate sub-sampling.

\subsection{Full patient group}

In general, the higher the frame rate of data used, the higher the velocity measured, as shown in the full datasets of all patients in Figure 5a-c. Towards the left of the graph, which addresses progressively lower frame rates, the measured velocities decrease, ultimately tending towards zero at very low frame rates.

There are 25 patients each with 20 frame rates, so there are 500 black data points, with 36 absent data points for s' (Figure 5a), 97 absent data points for e' (Figure 5b) and 237 (140 due to atrial fibrillation (AF) patients) absent data points for a' (Figure 5c) at frame rates too low for the algorithm to work. The y coordinate was scaled so that the velocity obtained from the raw acquired image sequence (with no 1-in-n sub-sampling) appears as 1.0.

A summary of these results is shown in Figure 6, depicting the averages across all patients and a range given by a fit of $1-a e^{-b x}$ to the minimum and maximum mean values of patients. For s' waves (upper panel - Figure 6a), at frame rates above about 20 frames/s, the velocities measured were very close to those of the raw acquired data at 52 and 79 frames/s. For e' and 
a' waves (middle - Figure $6 \mathrm{~b}$ and lower - Figure 6c panels respectively), the velocities measured begin to drop when frame rate falls below about 40 frames/s.

\subsection{Dependence on heart rate}

In online appendix $\mathrm{A}$, these three curves are shown for the 25 patients ranked by heart rate, with the patient with the lowest heart rate shown first. There is no appreciable tendency for patients with higher heart rates to require higher frame rates to adequately detect peak velocities.

Figure 5 shows the graph of the measured velocity against frame rate of the data used, similar to Figure 3, but with the patients divided into below median heart rate (open circles) and above median heart rate (filled circles). The curves are similar.

\section{Discussion}

Speckle tracking is a widely used technique in echocardiographic image analysis for tracking patches of tissue from frame to frame throughout the image sequence and determining tissue velocity in the evaluation of left-ventricular function. It is known that reliable tracking of tissue velocities requires an appropriate frame rate. Too low a frame rate might result in speckles decorrelating and not being recognised in the next frame. Too high a frame rate might show virtually no movement of speckles between successive frames, again compromising velocity measurements. 
For speckle tracking analysis on echocardiographic images, it is commonly recommended to use acquisition frame rates between 40 to 80 frames/s [1, 2, 5-9].

However, there has been no in-depth quantitative report of the effects of high or low frame rates for speckle tracking. It is also not clear, to what extent patient heart rate affects what frame rates can be used for adequate speckle tracking.

The present study shows a reliable plateau for consistent velocity measurements above a frame rate of 40 frames/s, which provides a scientific and testable basis for current recommendations.

We found that when frame rate falls below 40 frames/s, peak velocities are no longer accurately identified. The different waves have different vulnerabilities to underestimation, because the duration of their peaks are different. A wave whose peak velocity is sustained for many milliseconds can be identified even from frames relatively far apart in time. In contrast if a wave has only very brief peak, with substantially lower velocities immediately before and after, then widely spaced frames can only capture the average velocity during the interval between them and this will be a mixture of the peak velocity and the lower velocities before and after. As a result the peak velocities will be underestimated. In our study we found that the s' wave, whose peak is longer in duration, can have accurate velocity measurements down to a frame rate of about 20 frames/s. However A' and E' waves have narrower more pointed peaks, and require frame rates above about 40 frames/s. 
Figure 4 shows this graphically in the velocity traces of one patient. Figure 3 shows progressive decline in measured peak velocities as frame rate is dropped below a threshold, which is lower for $S^{\prime}$ than for E' and $A^{\prime}$. The same general pattern is also found in all patients as shown in online appendix A, and summarized in Figure 5. This appears to not to have been noted in speckle tracking, although a parallel phenomenon has been described in tissue Doppler [11, 12].

One of the 25 patients showed data that did not fit the general pattern described above. In online appendix $\mathrm{C}$ this patient is separated away (right panel) from the remaining 24 patients (left panel). For unclear reasons many of the frame rates below the raw acquired data at 52 and 79 frame/s showed higher velocities. We have uploaded the raw 52 and 79 frames/s data of this patient to assist other workers studying this field.

We were surprised to find, that patients with atrial fibrillation (online appendix A) did not have a dramatically different pattern from those in sinus rhythm. We believe the reason for this is the large number of beats we acquired, measured and averaged, which was over 20 beats per acquired frame rate.

Appendix D also provides the Matlab code used in this analysis to help anyone test on their image sequences the frame rate at which the patients need to be scanned. The code welcomes improvements.

\section{Study Limitations}


The results from this study have been obtained from a relatively small number of patients. However they were unselected outpatients that are representative of those who attend cardiology clinics.

We only acquired raw data at two frame rates (52 and 79 frames/s). The other frame rates were obtained by sub-sampling. It is theoretically possible that different results would have been found, if we had directly acquired at the lower frame rates rather deriving these by subsampling. However, if every time we make a fresh acquisition at a different frame rate, there is chance for biological variability to alter fine details of cardiac velocity and exact tissue planes. Sub-sampling ensured that, at least within each set of 10 frame rates derived from a single raw acquired frame rate, exactly the same image data would be used.

All image sequences were obtained with a constant image resolution of $640 \times 480$ pixels. Higher or lower image resolutions might give different results.

Moreover we only used our own speckle tracking algorithm and not any commercial algorithm governed by secrecy rules. Other algorithms might give different results. However only openly readable and editable algorithms are amenable to scientific research on the causes and remediation of inaccuracies, since parts can be changes the effects assessed. For example we have applied a particular set of settings. Any reader can modify our software and test whether alternative settings give different results. Readers are also free to compare our algorithms (without needing to seek permission) to closed source commercial algorithms (if they can acquire permission). 
For example when pieces of one frame are compared with approximately similarly located pieces of the next frame, it matters how far away the algorithm looks. If it looks only short distance, it will be unable to track tissue that is moving very fast. If it looks too long a distance, it might capture erroneous velocities because of false positive matching to unrelated tissue far away, and will be very slow to run as it will have to make many more comparisons.

All the patients in our study were at rest. During stress tests, heart rate is higher by two to three fold. Results might be different in such states, because the individual waves would be necessarily shorter in duration and therefore the frame rates required for adequate velocity tracking might be higher. All that we can say, is that across the $39 \mathrm{bpm}$ to $124 \mathrm{bpm}$ range of heart rates that we studied, there was no detectable tendency for the higher heart rates to require higher frame rates for adequate velocity measurement.

\section{Conclusions}

This study provides quantitative data showing that appropriate frame rate is essential for correct velocity measurement by speckle tracking echocardiography. A frame rate of at least 40 frames /s is necessary and sufficient, with a marked reduction in measured e' and a' velocities at lower frame rates. The s' wave is less vulnerable to underestimation and can generally be measured correctly down to frame rates of about 20 frames/s. Atrial fibrillation patients do not show any 
different requirement for frame rate. Across the $39 \mathrm{bpm}$ to $124 \mathrm{bpm}$ range of heart rates of patients in our study, we saw no tendency for higher heart rates to require higher frame rates. Attached to this article, we provide our vendor-independent software used in the analysis. Readers are free to edit or adapt it without needing permission, and can test it against commercial algorithms, if permission can be obtained.

\section{Acknowledgements}

The authors are grateful to the European Research Council and the British Heart Foundation for providing funding support for this study. M.N., M.Z., N.D., and D.F. were funded by the ERC (281524). G.C. was funded by the BHF (FS/12/12/29294). M.M. was supported by a Junior Research Fellowship at Imperial College London. 


\section{References}

[1].S. Mondillo, M. Galderisi, D. Mele, et al., Speckle-tracking echocardiography: a new technique for assessing myocardial function, J Ultrasound Med 30(1) (2011) 71-83

[2]. H. Blessberger, T. Binder, Non-invasive imaging: two dimensional speckle tracking echocardiography_basic principles, Heart 96 (2010) 716-722

[3].H. Geyer, G. Caracciolo, H. Abe, et al., Assessment of myocardial mechanics using speckle tracking echocardiography: fundamentals and clinical applications, J Am Soc Echocardiogr 23(4) (2010) 351-69

[4]. M.C. Donadio Abduch, A. Mesquita Alencar, W. Mathias, M. Luiz de Campos Vieira, Cardiac Mechanics Evaluated by Speckle Tracking Echocardiography, Arq Bras Cardiol 102(4) (2014) 403-412

[5].A. Rosner, D. Barbosa, E. Aarsæther, D. Kjønas, H. Schirmer, J. D’hooge, The influence of frame rate on two-dimensional speckle-tracking strain measurements: a study on silico-simulated models and images recorded in patients, Eur Heart J Cardiovasc Imaging 16 (2015) 1137-1147

[6].A.A. Sanchez, P.T. Levy, T.J. Sekarski, A. Hamvas, M.R. Holland, G.K. Singh, Effects of Frame Rate on Two-Dimensional Speckle Tracking-Derived Measurements of Myocardial Deformation in Premature Infants, Echocardiography 00 (2014) 1-9 
[7].G.K. Singh, B. Cupps, M. Pasque, P.K. Woodard, M.R. Holland, A. Ludomirsky, Accuracy and reproducibility of strain by speckle tracking in pediatric subjects with normal heart and single ventricular physiology: a two-dimensional speckle-tracking echocardiography and magnetic resonance imaging correlative study, J Am Soc Echocardiogr 23(11) (2010) 1143-52

[8].J.-U. Voigt, G. Pedrizzetti, P. Lysyansky, et al., Definitions for a Common Standard for 2D Speckle Tracking Echocardiography: Consensus Document of the EACVI/ASE/Industry Task Force to Standardize Deformation Imaging, Eur Heart J Cardiovasc Imaging 16(1) (2015) 1-11

[9].V. Mor-Avi, R.M. Lang, L.P. Badano, et al., Current and evolving echocardiographic techniques for the quantitative evaluation of cardiac mechanics: ASE/EAE consensus statement on methodology and indications endorsed by the Japanese Society of Echocardiography, Eur J Echocardiogr 12(3) (2011) 167-205

[10]. M. Koestenberger, Transthoracic Echocardiography in Children and Young Adults with Congenital Heart Disease, ISRN Pediatr 2012 (2012) 753481

[11]. B. Lind, J. Nowark, J. Dorph, J. van der Linder, L.A. Brodin, Analysis of temporal requirements for myocardial tissue velocity imaging, Eur J Echocardiogr 3(3) (2002) 214-9

[12]. N. Elmstedt, B. Lind, K. Ferm-Widlund, M. Westgren, L.A. Brodin, Temporal frequency requirements for tissue velocity imaging of the fetal heart, Ultrasound Obstet Gynecol 38(4) (2011) 413-7 


\section{Figures:}
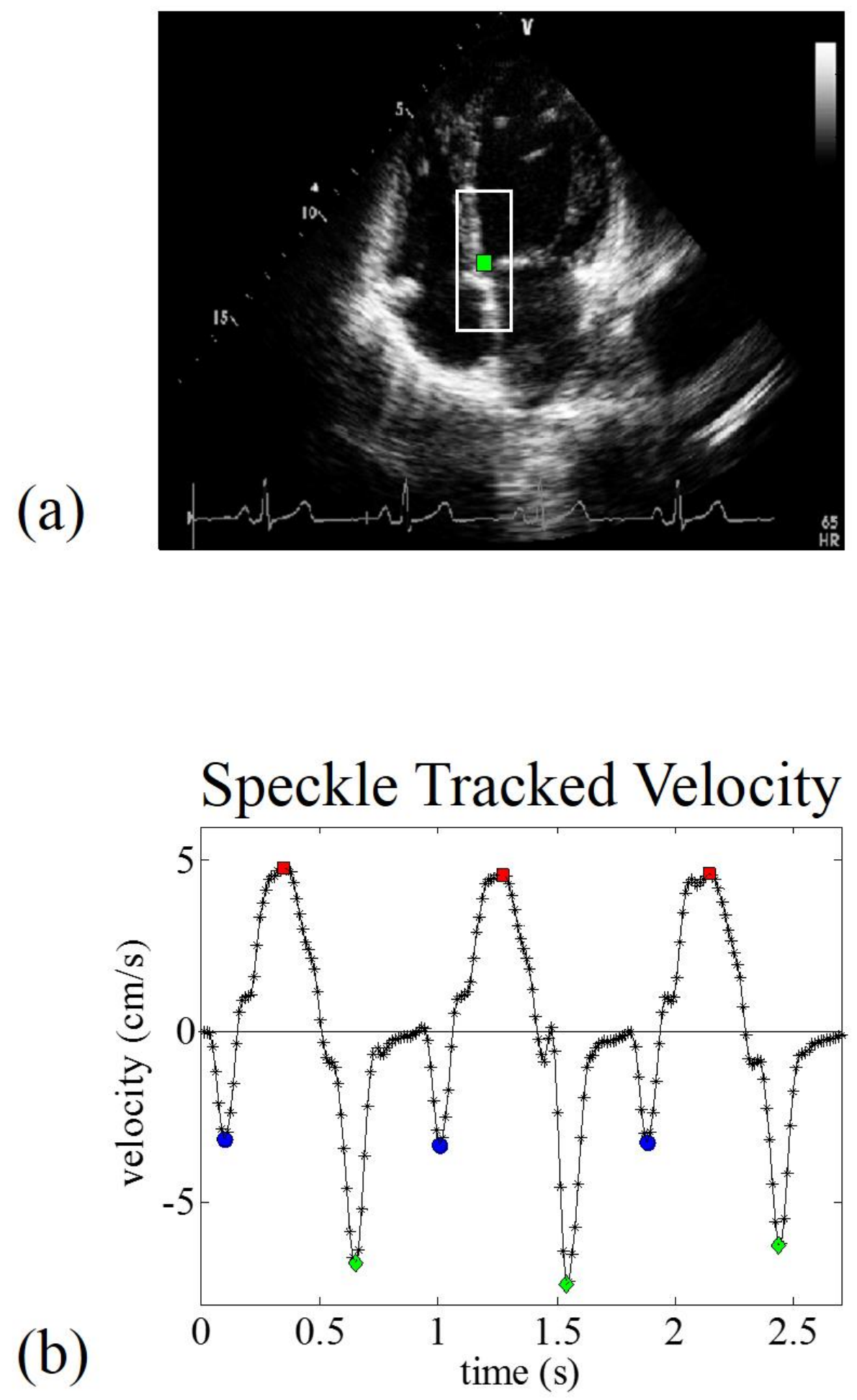

Fig. 1 (color). (a) Apical four chamber view echocardiographic image of the heart of an example patient used in the analysis, acquired at 52 frames/s. Speckle tracking was applied to the set of images acquired and the region of interest (ROI) was centred around the green point selected on the septal wall, i.e. the basal region of the wall. The white box was then used to extract tissue velocities. (b) Velocity curve for one example patient showing the selected peak velocities for systole (red squares $\bullet$ ), early (green diamonds $\diamond$ ) and late (blue circles $\bullet$ ) diastole. 

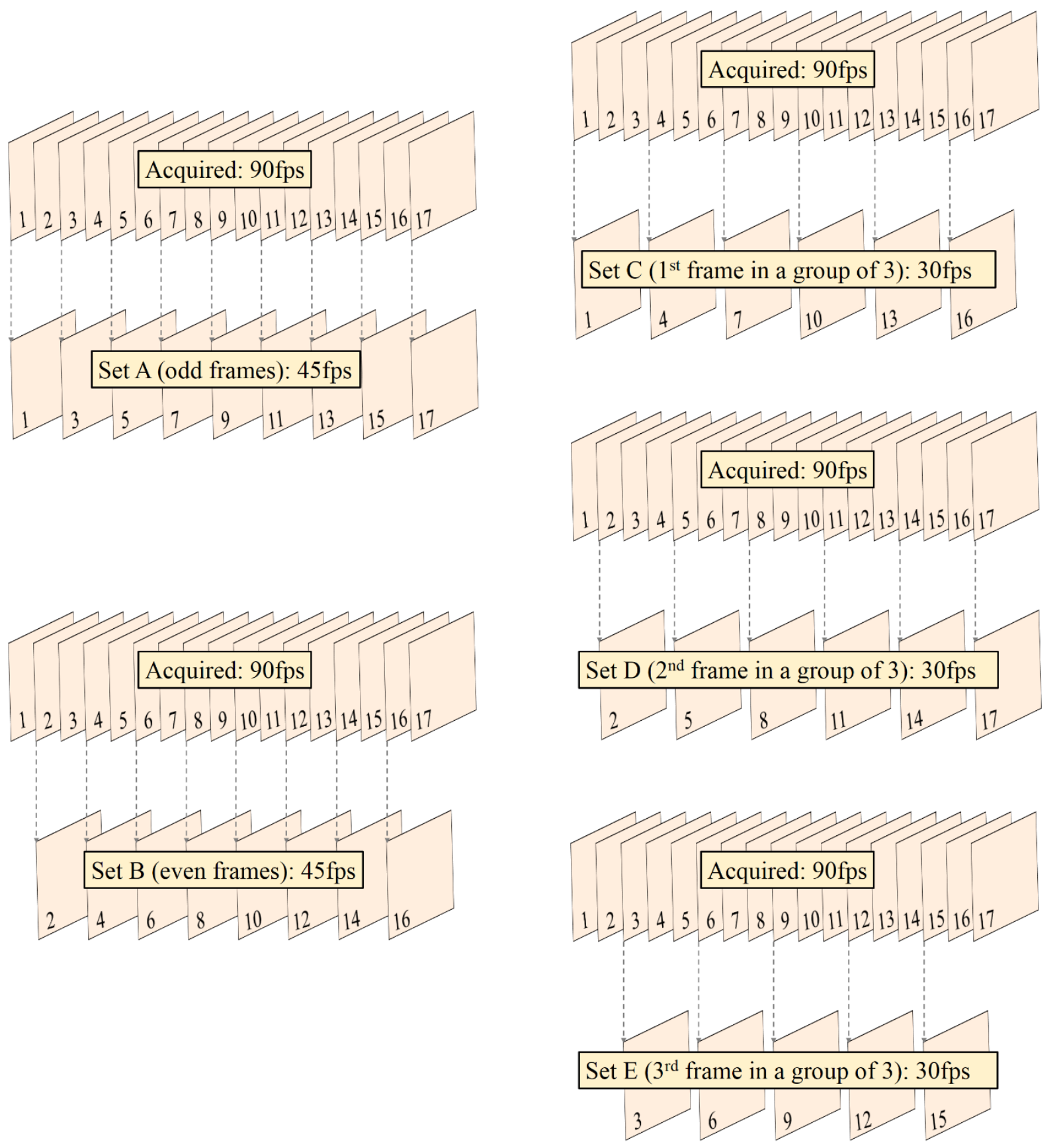

Fig. 2 (color). Schematic of the image sequences used in the analysis as obtained from dropping frames from the acquired sequence. First, every other frame is dropped, left top (only odd frames are retained) and left bottom (only even frames are retained) for analysis. Then, from the acquired image sequence right top (one frame in three is retained), right middle (the second frame in every three frames is retained) and right bottom (the third frame in every group of three frames is retained). The frame rate is decreased further up to keeping one frame in a group of ten, resulting ten loops for a sampling frequency ten times smaller than acquired original frame rate. 


\section{Source Data at $79 \mathrm{fps}$ and $52 \mathrm{fps}$ and Data Sets Created from Them}

(a)

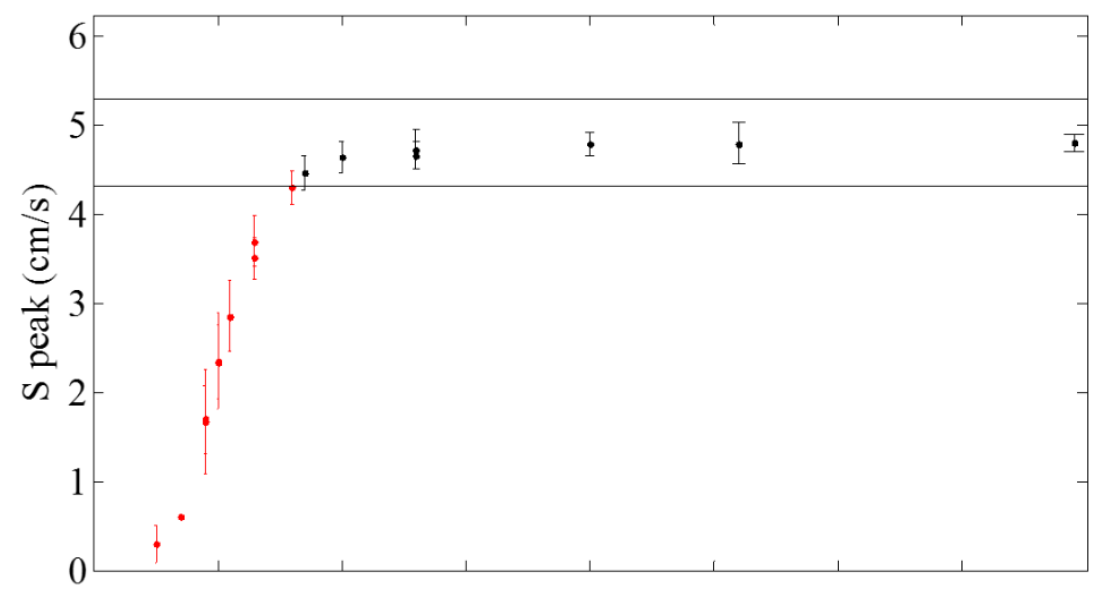

(b)

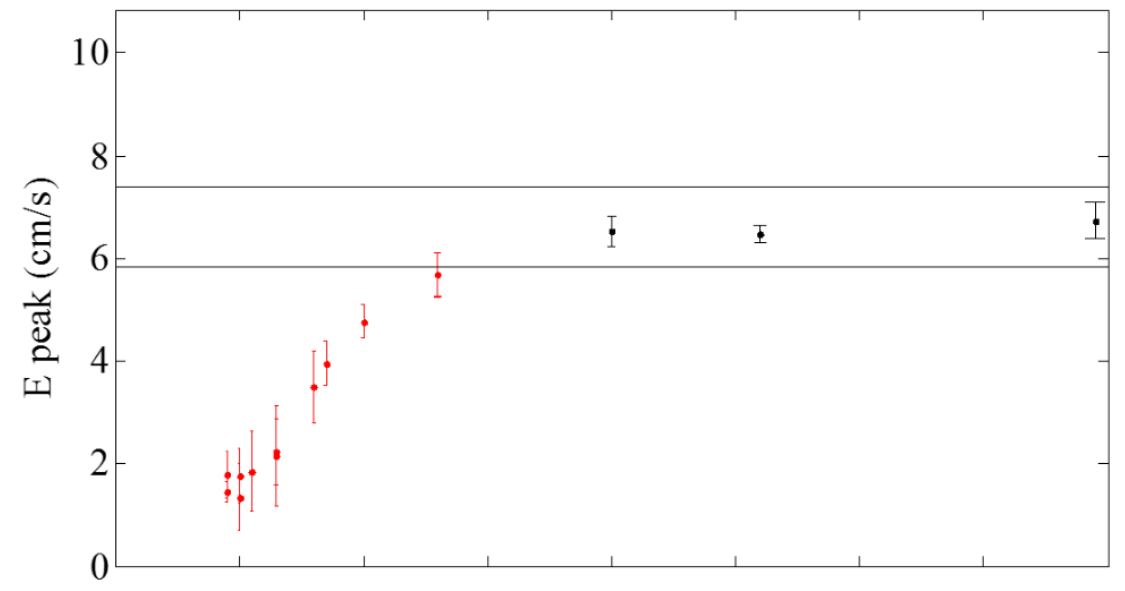

(c)

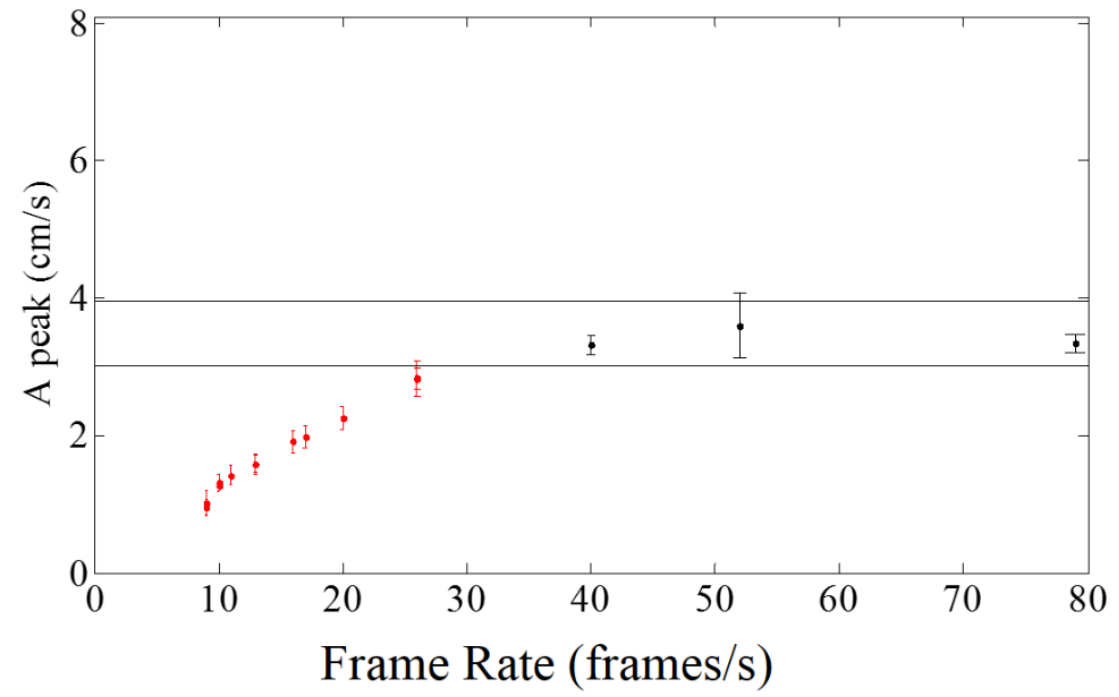

Fig. 3 (color). Relationship between peak systolic (a), early (b) and late (c) diastolic velocities vs. frame rate for one example patient. The image sequences are acquired at $52 \mathrm{fps}$ and $79 \mathrm{fps}$. The black points $(\bullet)$ are the mean values for frames dropped from the acquired image sequences and the errorbars are the standard deviation corresponding to that frequency. For each frame rate where frames are dropped, the plotted values are calculated as mean of all peak values in all sequences. 
Velocity curve smoothed with Savitsky-Golay filter of $3^{\text {rd }}$

polynomial degree and 11 points for extracting S peak points

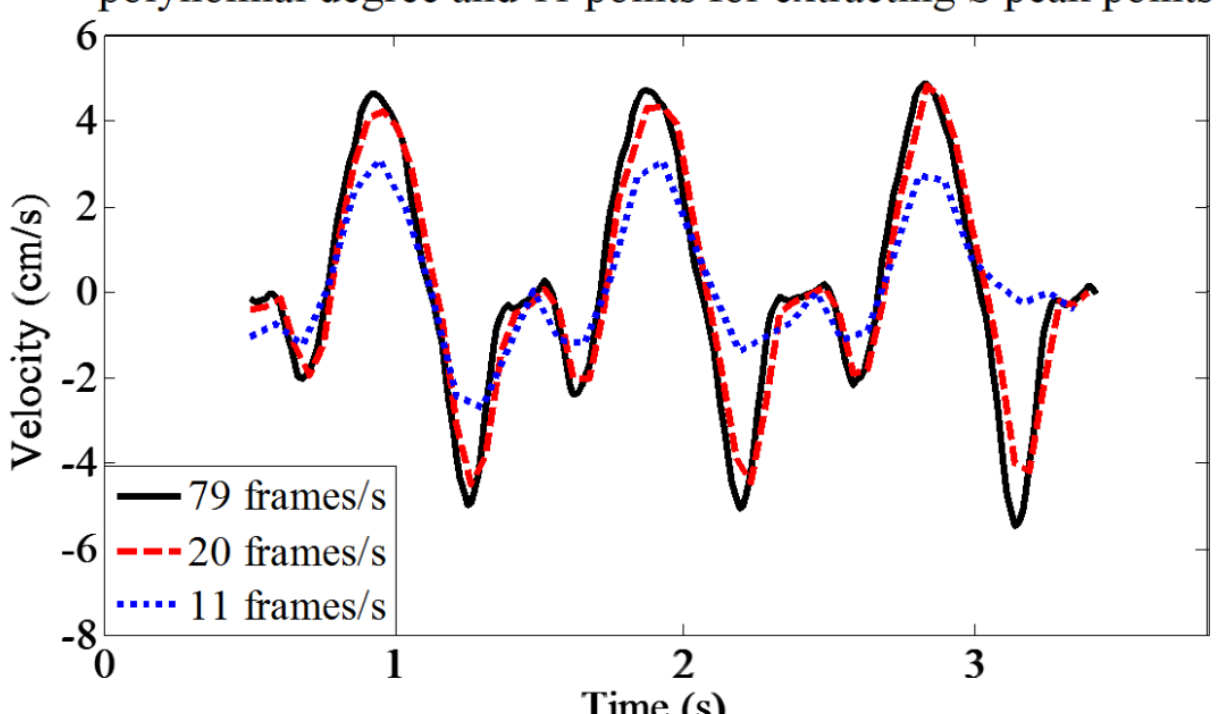

(a)

Time (s)

Velocity curve smoothed with Savitsky-Golay filter of $5^{\text {th }}$ polynomial degree and 7 points for extracting $E$ and A peak points

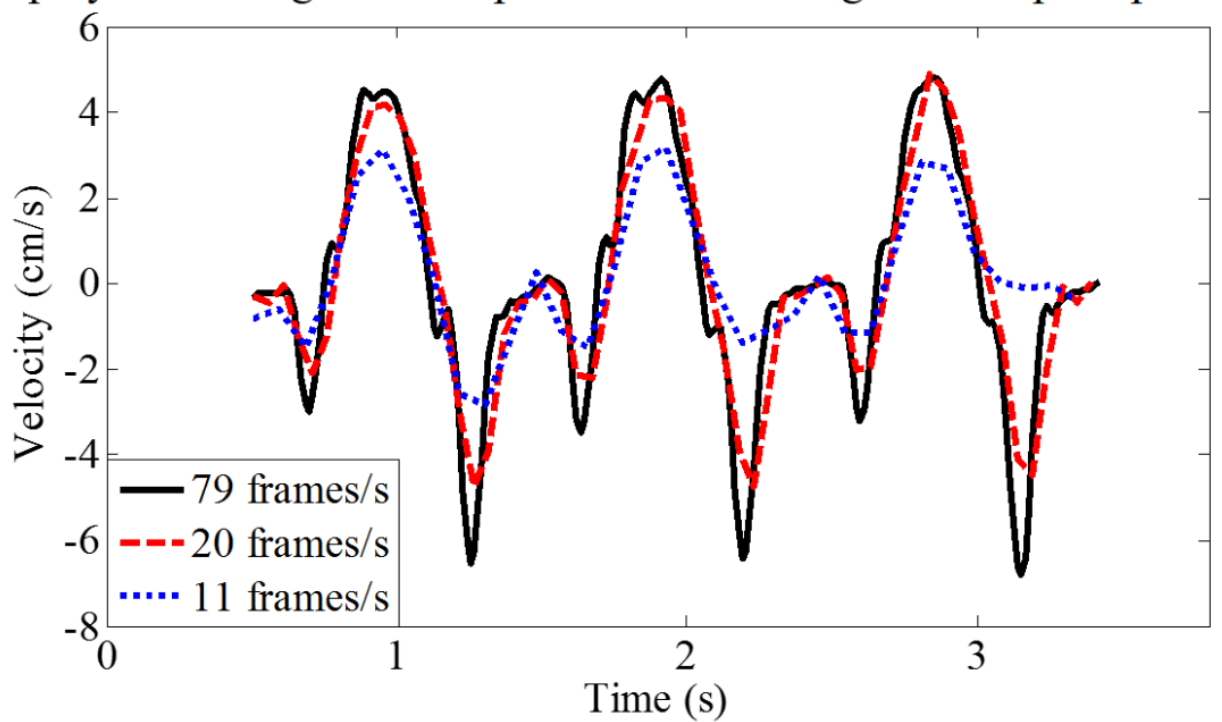

(b)

Time (s)

Fig. 4 (color). (a) Velocity curves calculated from speckle tracking on preceding frame to frame for one patient, for images acquired at $79 \mathrm{fps}$ (black - - ), 25\% of acquired images (20 fps, red - -) and 14\% of acquired images (11 fps blue $\cdot-$ ) for velocity curves smoothed with a Savitsky-Golay filter of $3^{\text {rd }}$ polynomial degree and 11 points used for extracting s' wave. (b) Velocity curves calculated from speckle tracking on preceding frame to frame for one patient, for images acquired at $79 \mathrm{fps}$ (black - ), 25\% of acquired images (20 fps, red $-\longrightarrow)$ and $14 \%$ of acquired images (11 fps blue -*) for velocity curves smoothed with a Savitsky-Golay filter of $5^{\text {th }}$ polynomial degree and 7 points used for extracting e' and a' waves. 
(a)

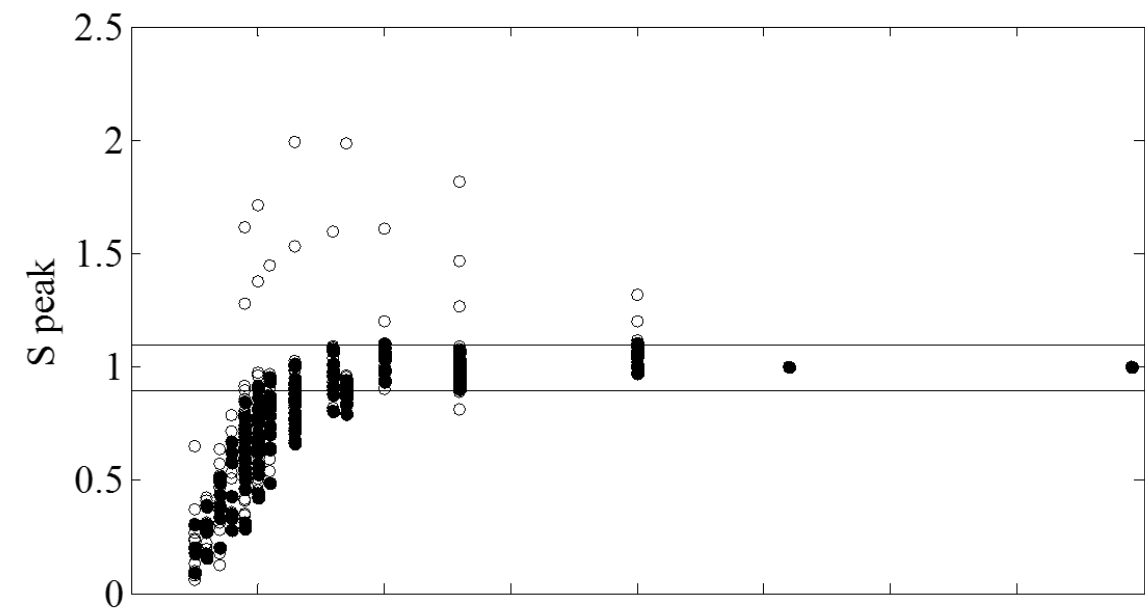

(b)
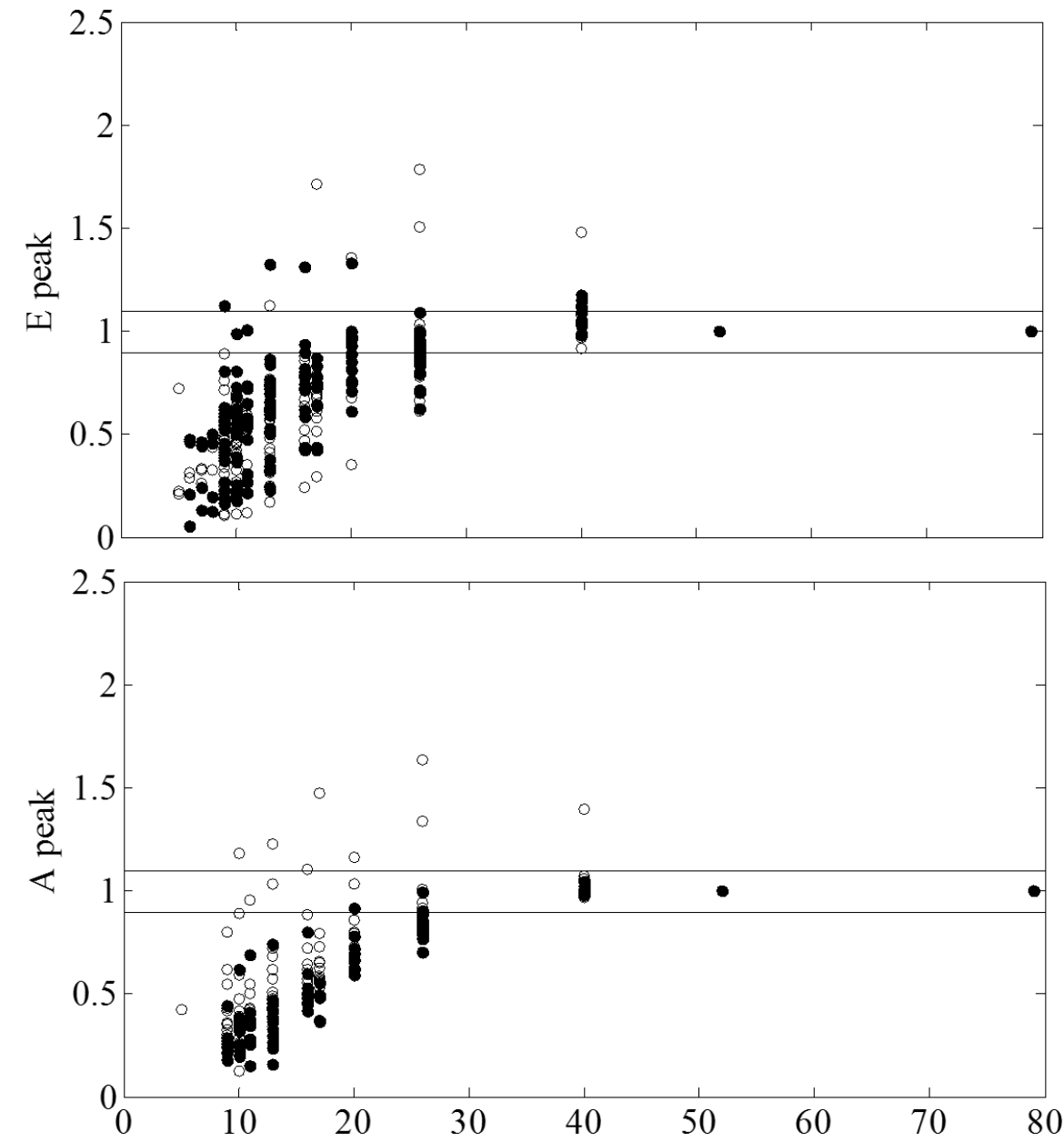

Frame Rate (frames/s)

Fig. 5 (color). Relationship between peak velocities and frame rate for peak systolic velocity (a) and early (b) and late (c) peak diastolic velocities, for 25 patients. The circles represent the mean peak velocities for the acquired and dropped image sequences (open circles represent patients with heart rate below median while filled circles, patients with heart rate above median). Velocities where image sequences are dropped, are normalised to the corresponding mean acquired velocity. The images were acquired at $52 \mathrm{fps}$ and $79 \mathrm{fps}$ on the patients' basic 
(a)

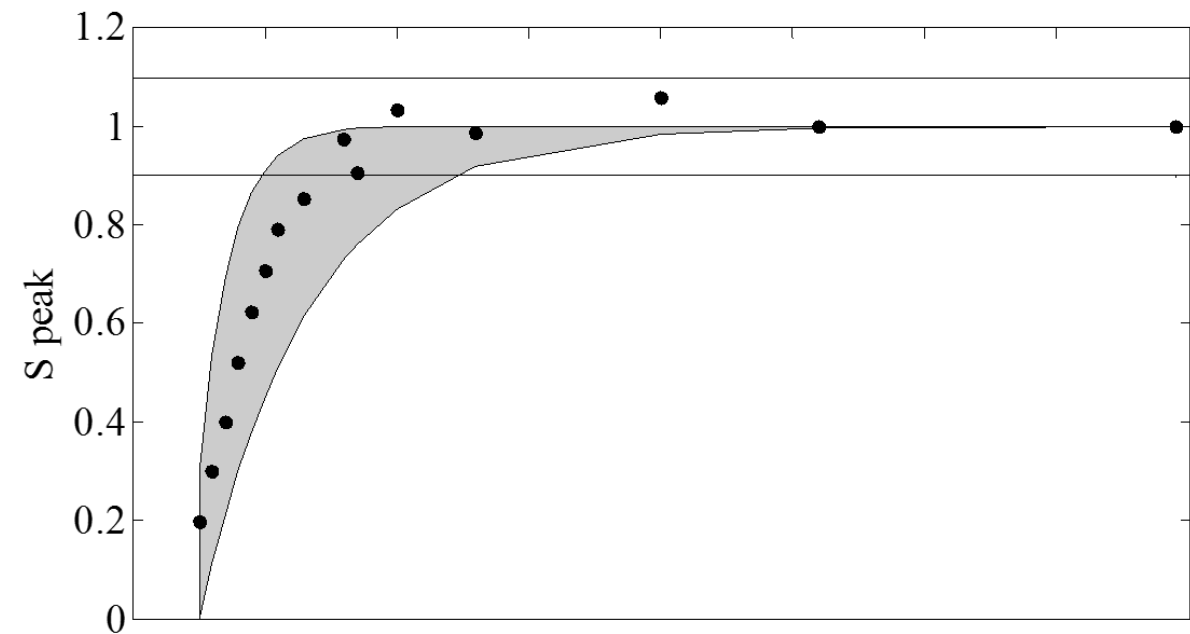

(b)

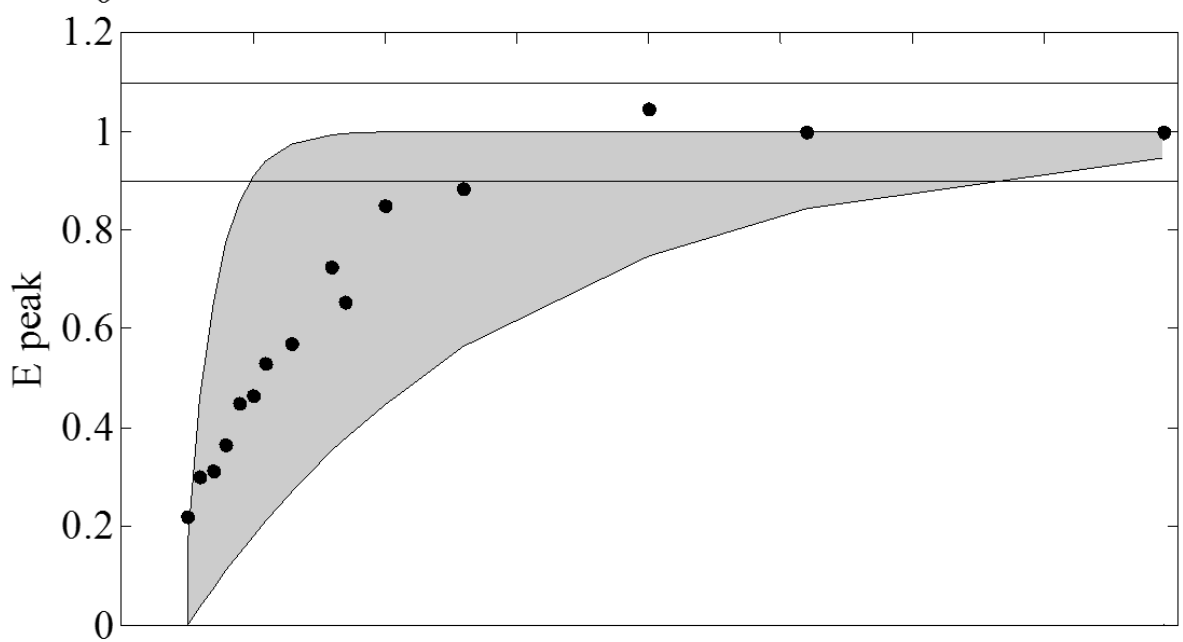

(c)

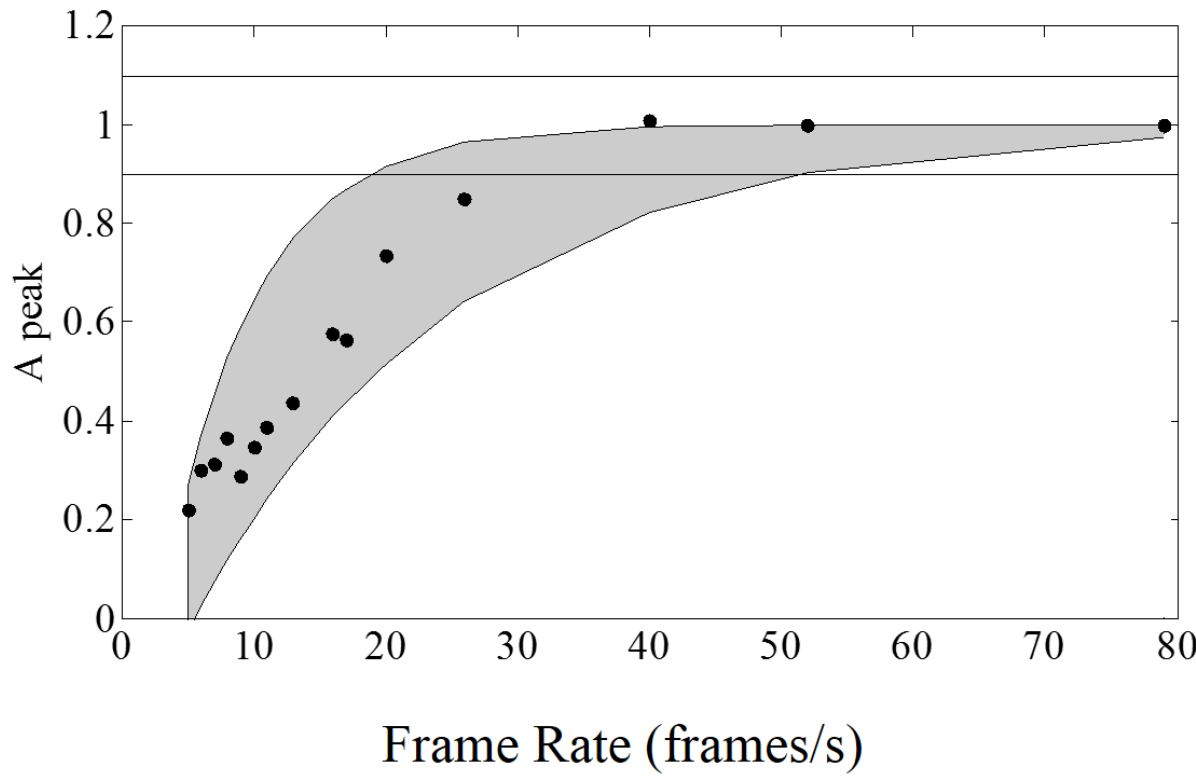

Fig. 6 (color). Summary of Figure 5 showing the averages across all patients for each frame rate as black circles.

The range is given by fitting the minimum or maximum values with a function of $1-a e^{-b x}$. For s' waves (a) consistent velocities are obtained above 20 frames/s, while for e' (b) and a' (c) waves, the velocities measured begin to drop when frame rate falls below 40 frames/s. 